\title{
COMPACTNESS THEOREMS FOR CRITICAL METRICS OF THE WEYL FUNCTIONAL ON COMPACT KÄHLER SURFACES
}

\author{
SHUN-CHENG CHANG
}

(Received 27 January 1994)

Communicated by R. Bartnik

\begin{abstract}
In this note, we propose an extension of the compactness property for Kähler-Einstein metrics to critical metrics of Weyl functional on compact Kähler surfaces.
\end{abstract}

1991 Mathematics subject classification (Amer. Math. Soc.): primary 53C25, 58G30, 53C20.

\section{Introduction}

We will consider the following quadratic Weyl functional

$$
\mathrm{SW}(g)=\int_{M}\left|W_{i j k l}\right|^{2} d \mu_{g}
$$

on a closed Kähler 4-manifold $M$. The critical metrics on $M$ are defined to be the critical points of SW. Then all metrics which are conformal to Einstein metrics are critical metrics. In particular, all Kähler-Einstein metrics on $M$ are critical metrics for SW. On the other hand, Tian proved that the space of Kähler-Einstein metrics on a compact Kähler surface $M$ has a certain compactness property $([8,9])$. In this article, we will give some results about the compactness of certain critical metrics of SW on $M$ which are served as a generalization of Tian's results on compactness of Kähler-Einstein manifolds. Our results read:

THEOREM 1.1. Let $W(M)$ be the set of isometry classes of all smooth critical metrics of SW on a compact Kähler 4-manifold $M$ such that 

(i) $\int_{M}|\mathrm{Rc}|^{2} d \mu \leq H$,
(ii) $\operatorname{Rc}(g) \geq-K g$,
(iii) $i_{M} \geq i_{0}>0$,
(iv) $\operatorname{diam}_{M} \leq d$,

for fixed positive constants $H, K, d, i_{0}$. Then $W(M)$ is compact in the $C^{\infty}$-topology.

Furthermore, we have

THEOREM 1.2. Let $\widetilde{W}(M)$ be the set of isometry classes of all smooth critical metrics of SW on a compact Kähler 4-manifold $M$ such that

(i) $\int_{M}|\mathrm{Rc}|^{2} d \mu \leq H$,

(ii) $\operatorname{diam}\left(S_{\rho}\right) \leq d \rho$,

(iii) $i_{M} \geq i_{0}>0$,

for fixed positive constants $H, d, i_{0}$. Here $S_{\rho}$ is the geodesic sphere.

Then $\widetilde{W}(M)$ is compact in the $C^{\infty}$-topology.

On the other hand, if we replace the lower bound on the injectivity radius by a local volume bound, and then follow Tian's arguments as in [8], we obtain

THEOREM 1.3. For any positive constant $H, K, d, V$, let $\left\{\left(M_{i}, g^{i}\right)\right\}$ be a sequence of compact critical Kähler 4-manifolds satisfying

(i) $\int_{M}|\operatorname{Rc}|^{2} d \mu \leq H$,

(ii) $\operatorname{Rc}(g) \geq-K g$,

(iii) $\operatorname{Vol}(M) \geq V$,

(iv) $\operatorname{diam}_{M} \leq d$.

Then a subsequence of $\left\{\left(M_{i}, g^{i}\right)\right\}$ converges to a critical Kähler 4 -orbifold $\left\{\left(M_{\infty}, g^{\infty}\right)\right\}$ with finitely many isolated singular points $S_{\infty}$. More precisely, we may take a subsequence of $\left\{\left(M_{i}, g^{i}\right)\right\}$, say $\left\{\left(M_{i} \backslash S_{i}, g^{i}\right)\right\}$ for $S_{i}=\left\{x_{i 1}, x_{i N}\right\}$, which converges to a critical Kähler surface $\left\{\left(M_{\infty} \backslash S_{\infty}, g^{\infty}\right)\right\}$ in the following sense: for any compact subset $K \subset M_{\infty} \backslash S_{\infty}$, there exists an $r>0$ such that there exist diffeomorphisms $\phi_{i}$ from $M_{i} \backslash \bigcup_{j=1}^{N} B_{r}\left(x_{i j}\right)$ into $M_{\infty} \backslash S_{\infty}$ such that

(v) $\left(\phi_{i}^{-1}\right)^{*} g^{i}$ converges to $g^{\infty}$ uniformly on $K$,

(vi) $\left(\phi_{i}\right)_{*} \circ J_{i} \circ\left(\phi_{i}^{-1}\right)_{*}$ converges to $J_{\infty}$ uniformly on $K$, where $J_{i}, J_{\infty}$ are the almost complex structures of $M_{i}, M_{\infty}$, respectively.

Moreover, we have

THEOREM 1.4. For any positive constant $H, d$, let $\left\{\left(M_{i}, g^{i}\right)\right\}$ be a sequence of compact critical Kähler 4-manifolds satisfying

(i) $\int_{M}|\operatorname{Rc}|^{2} d \mu \leq H$, 
(ii) there is a local Sobolev constant $C_{s}$ bound, that is, for fuxed small $\rho>0$

$$
\|f\|_{4}^{2} \leq C_{s}\|\nabla f\|_{2}^{2}, \quad f \in C_{0}^{\infty}\left(B_{\rho}\right),
$$

(iii) $\operatorname{diam}\left(S_{\rho}\right) \leq d \rho$,

then a subsequence of $\left\{\left(M_{i}, g^{i}\right)\right\}$ converges to a critical Kähler 4-orbifold with finitely many isolated singular points in the sense of Theorem 1.3.

The organization of this article is as follows: In Section 2, we use critical equations as in [2] to obtain local curvature estimates. In Section 3, based on the hypothesis (ii) of Theorem 1.1 and Lemma 3.1 and the volume growth property of a small geodesic ball, we give a diameter estimate on this geodesic sphere ([5]). Finally, in Section 4, we study the tangent cone metric by a blowing up argument; this and the diameter estimate yield that this tangent cone metric is the flat Euclidean metric (cf. [9]), and we then obtain the maximal volume growth property. Thus we are able to apply results in [1] to finish the proof of our Theorem 1.1 and Theorem 1.2 under the lower bound of injectivity radius, and the arguments as in $[8,9]$ to complete the proof of our Theorem 1.3 and Theorem 1.4.

\section{Critical equations and local curvature estimates on Kähler surfaces}

For a critical metric $g$ of SW on a compact Kähler surface $M$, the Bach tensor must be identically zero, that is, $B(g)=0$. Then we have ([3])

LEMMA 2.1. On a Kähler surface $(M, g)$, the following are equivalent:

(i) $B(g)=0$,

(ii) $2 \nabla_{i} \nabla_{j} R=\left(R^{2} / 4+\triangle R / 2\right) g_{i j}-R R_{i j}$, where $R$ is the scalar curvature of $g$.

From Lemma 2.1, we have the following critical equations on the compact Kähler surface $M$ :

LEMMA 2.2. For a critical metric $g$ of $\mathrm{SW}$ on $M$, the Ricci curvature tensor $\boldsymbol{R}_{i j}$ satisfies the following:

$$
\frac{1}{2} \Delta R_{i j}-\left(\frac{R^{2}}{2}+\Delta R\right) g_{i j}+2 R R_{i j}-R_{i}^{p}{ }_{i}^{k}{ }_{j} R_{p k}-\frac{1}{4}|\mathrm{Rc}|^{2} g_{i j}=0 .
$$

PROOF. From [3], we have the Bach tensor

$$
B_{i j}=\nabla^{p} \nabla^{q} W_{p i j q}+\frac{1}{2} R^{p q} W_{p i j q}=0 .
$$


Now the second Bianchi identity implies the following divergence formulae:

$$
\begin{aligned}
-\nabla^{p} R_{p i j k} & =\nabla_{k} R_{i j}-\nabla_{j} R_{i k} \\
-\nabla^{p} R_{i p} & =-\frac{1}{2} \nabla_{i} R \\
-\nabla^{p} W_{p i j k} & =\nabla_{k} P_{i j}-\nabla_{j} P_{i k},
\end{aligned}
$$

where $P_{i j}=(n-3)\left[R_{i j}-R g_{i j} /(2 n-2)\right] /(n-2)$. Then, for $n=4$,

$$
12 \nabla^{p} \nabla^{q} W_{p k j q}=6 \nabla^{p} \nabla_{k} R_{j p}-6 \nabla^{p} \nabla_{p} R_{k j}-\nabla_{k} \nabla_{j} R+\Delta R g_{k j} .
$$

On the other hand, (1) and the Ricci identity imply

$$
\nabla^{p} \nabla_{k} R_{j p}=\nabla^{p} \nabla_{j} R_{k p}
$$

This implies

$$
\begin{aligned}
B_{i j}= & \nabla^{p} \nabla_{j} R_{i p}-\frac{1}{2} \Delta R_{i j}-R^{p}{ }_{i} R_{p j}+\frac{1}{4}|\mathrm{Rc}|^{2} g_{i j} \\
& +\frac{R}{3} R_{i j}-\frac{1}{3} \nabla_{j} \nabla_{i} R+\frac{1}{12} \Delta R g_{i j}-\frac{1}{12} R^{2} g_{i j} \\
= & 0 .
\end{aligned}
$$

This and Lemma 2.1 imply Lemma 2.2 .

COROLLARY 2.3. For any critical metric $g$ of $\mathrm{SW}$ on $\mathrm{M}$, we have

$$
\Delta \mathrm{Rc}+\mathrm{Rm} * \mathrm{Rc}=0,
$$

where we write $A * B$ for any linear combination of tensors $A, B$ by contraction using $g^{i j}$.

From Corollary 2.3, for the critical metric $g_{i j}$ of $\mathrm{SW}$ on a Kähler surface $\mathrm{M}$, we get

LEMMA 2.4. There exists a constant $C>0$ such that $-\Delta f \leq b f$, where $f=$ $|\mathrm{Rc}|, b=C|\mathrm{Rm}|$.

Furthermore, for higher order derivatives of the critical metric, we have

LEMMA 2.5. For any positive integer $l$ and the critical metric $g$ on $M$, we obtain

$$
\Delta\left(\nabla^{l} \mathrm{Rc}\right)=\sum_{k=0}^{l} \nabla^{k} \mathrm{Rm} * \nabla^{l-k} \mathrm{Rc} .
$$


Now we are ready to get the local curvature estimates. Let $B_{\rho}\left(x_{0}\right)$ be a geodesic ball in $\mathbf{M}$ with center $x_{0}$ such that the local Sobolev constant $C_{s}$ bound, that is,

$$
\|f\|_{4}^{2} \leq C_{s}\|\nabla f\|_{2}^{2}, \quad f \in C_{0}^{\infty}\left(B_{\rho}\left(x_{0}\right)\right) .
$$

Then, from [2], based on Lemma 2.4 and (2), integration by parts and Moser iteration, we have

PROPOSITION 2.6. ([2, Theorem 3.3.]) For any critical metric $g$ on $\mathbf{M}$ and any $0<\eta<1$, there exists $\kappa_{1}>0$ such that, if $\int_{B_{\theta}}|\mathrm{Rm}|^{2} d \mu \leq \kappa_{1}$, then

$$
\sup _{B_{(1-\eta) \rho}}|\operatorname{Rc}| \leq C\left(\rho, \eta, \kappa_{1}, C_{s}\right), \quad l=0,1,2, \ldots
$$

ProOF. We will sketch the proof; for details, see [2]. Define $\phi_{\rho}:[0, \rho] \mapsto[0,1]$ to be a $C^{\infty}$-function given by

$$
\phi_{\rho}(t)=\phi(t / \rho), \quad \phi_{\rho}^{\prime} \leq 0, \quad\left|\phi_{\rho}^{\prime}\right| \leq c_{4}(\epsilon) / \rho .
$$

where $\phi:(-\infty, \infty) \mapsto[0,1]$ is a $C^{\infty}$-function with $\phi(t)=0$, for $t \geq 1, \phi(t)=1$ for $t \leq 1-\epsilon$.

Let $f=|\mathrm{Rc}|, B_{\rho}=B\left(x_{0}, \rho\right)$, and set $u(x)=\phi_{\rho}(r(x))$ and $r(x)=d\left(x, x_{0}\right)$. We may assume $f>0$, take $p \geq 1$, and $u^{2} f^{2 p-1}$ as a test function; then, integrating by parts, one obtains

$$
\begin{aligned}
\int_{B_{\rho}}\langle\Delta & \left.f, u^{2} f^{2 p-1}\right\rangle d \mu=-\int_{B_{\rho}}\left\langle\nabla f, \nabla\left(u^{2} f^{2 p-1}\right)\right\rangle d \mu \\
= & (p-1) / 2 p^{2} \int_{B_{\rho}}\left\langle\nabla u^{2}, \nabla f^{2 p}\right\rangle d \mu-(2 p-1) / p^{2} \int_{B_{\rho}}\left|\nabla\left(u f^{p}\right)\right|^{2} d \mu \\
& +(2 p-1) / p^{2} \int_{B_{\rho}}|\nabla u|^{2} f^{2 p} d \mu .
\end{aligned}
$$

Thus, combining Lemma 2.4 and Hölder's inequality, we obtain

$$
\begin{aligned}
\frac{2 p-1}{p^{2}} & \int_{B_{\rho}}\left|\nabla\left(u f^{p}\right)\right|^{2} d \mu \\
& \leq \int_{B_{\rho}} b u^{2} f^{2 p} d \mu+\frac{p-1}{2 p^{2}} \int_{B_{\rho}}\left\langle\nabla u^{2}, \nabla f^{2 p}\right\rangle d \mu+\frac{2 p-1}{p^{2}} \int_{B_{\rho}}|\nabla u|^{2} f^{2 p} d \mu \\
& \leq \int_{B_{\rho}} b u^{2} f^{2 p} d \mu-\frac{p-1}{2 p^{2}} \int_{B_{\rho}} u^{2} \Delta f^{2 p} d \mu+\frac{2 p-1}{p^{2}} \int_{B_{\rho}}|\nabla u|^{2} f^{2 p} d \mu \\
& \leq \frac{2 p-1}{p} \int_{B_{\rho}} b u^{2} f^{2 p} d \mu+\frac{2 p-1}{p^{2}} \int_{B_{\rho}}|\nabla u|^{2} f^{2 p} d \mu \\
& \leq \frac{2 p-1}{p}\left(\int_{B_{\rho}} b^{2} d \mu\right)^{1 / 2}\left(\int_{B_{\rho}}\left(u f^{p}\right)^{4} d \mu\right)^{1 / 2}+\frac{2 p-1}{p^{2}} \int_{B_{\rho}}|\nabla u|^{2} f^{2 p} d \mu .
\end{aligned}
$$


This implies

$$
\int_{B_{\rho}}\left|\nabla\left(u f^{p}\right)\right|^{2} d \mu \leq p\left(\int_{B_{\rho}} b^{2} d \mu\right)^{1 / 2}\left(\int_{B_{\rho}}\left(u f^{p}\right)^{4} d \mu\right)^{1 / 2}+\int_{B_{\rho}}|\nabla u|^{2} f^{2 p} d \mu .
$$

We have the following Sobolev inequality for $n=4$ :

$$
\left(\int_{B_{\rho}}\left(u f^{p}\right)^{4} d \mu\right)^{1 / 2} \leq C \int_{B_{\rho}}\left|\nabla\left(u f^{p}\right)\right|^{2} d \mu .
$$

Then

$$
\left(\int_{B_{\rho}}\left(u f^{p}\right)^{4} d \mu\right)^{1 / 2} \leq C p\left(\int_{B_{\rho}} b^{2} d \mu\right)^{1 / 2}\left(\int_{B_{\rho}}\left(u f^{p}\right)^{4} d \mu\right)^{1 / 2}+\frac{C}{\rho^{2}} \int_{B_{\rho}} f^{2 p} d \mu .
$$

If $\left(\int_{B_{\rho}} b^{2} d \mu\right)^{1 / 2}$ is small, then we have

$$
\left(\int_{B_{(1-\epsilon)^{2} \rho}}\left(f^{p}\right)^{4} d \mu\right)^{1 / 2} \leq C \int_{B_{\rho}} f^{2 p} d \mu
$$

Set $(1-\epsilon)^{2}=1-\eta$; then

$$
\left(\int_{B_{(1-n) \rho}}\left(f^{p}\right)^{4} d \mu\right)^{1 / 4} \leq C\left(\int_{B_{\rho}} f^{2 p} d \mu\right)^{1 / 2}
$$

For $p=1$, we have

$$
\left(\int_{B_{(1-\eta) \rho}} f^{4} d \mu\right)^{1 / 4} \leq C\left(\int_{B_{\rho}} f^{2} d \mu\right)^{1 / 2}
$$

Now using Moser iteration, we obtain

$$
\sup _{B\left(x_{0},(1-\eta) \rho\right)}|\mathrm{Rc}| \leq C\left(\rho, \eta, \kappa_{1}, C_{s}\right) .
$$

In order to estimate the higher order covariant derivative of Ricci curvature tensor, we need the existence of harmonic coordinate on the ball with small $L^{2}$-norm of curvature.

LEMMA 2.7. ([6, Lemma 1.1.]) Given $0<\eta<1$, there exists a small constant $\kappa=$ $\kappa(H, V, D, n, \eta)>0$ such that, for any Riemannian manifold $(M, g)$ of dimension $n$, $x_{0} \in M$ with $|\operatorname{Rc}| \leq H, \operatorname{Vol}(M) \geq V, \operatorname{diam}(M) \leq D$,

$$
\int_{B\left(x_{0}, \rho(1+2 \eta)\right)}|\operatorname{Rm}|^{n / 2} d \mu \leq \kappa
$$


Then, for any $0<\delta<1 / 3$, and $x \in B\left(x_{0}, \rho(1+2 \eta)\right)$, so that $d\left(x_{0}, x\right)<1-\delta-2 \eta$, there exist harmonic coordinates $F$ with domain $D(F) \supset B(x, \delta \rho)$ and $\operatorname{Im} F \supset$ $B(\delta(1-\eta) \rho)=\left\{x \in R^{n}|| x \mid \leq \delta(1-\eta) \rho\right\}$, such that

(i) $F^{-1}(B(\delta(1-\eta) \rho)) \supset B(x, \delta(1-\eta) \rho)$.

Let $h_{i j}=\left\langle\nabla h_{i}, \nabla h_{j}\right\rangle$ be the metric tensor in such harmonic coordinates, $F=\left(h_{1}, \cdots, h_{n}\right), n \geq 4$.

(ii) $\left|h_{i j}-\delta_{i j}\right|_{C^{0}} \leq \eta^{2} / 100 n$.

(iii) $\left|d h_{i j}\right|_{C^{\alpha}} \leq C(H, V, D, n, \eta, \rho), \quad 0<\alpha<1$

(iv) $\left\|h_{i j}\right\|_{L^{2, p}} \leq C(H, V, D, n, \eta, \rho), \quad p>n$.

PROPOSITION 2.8. ([2, Theorem 3.9.]) For any critical metric $g$ on $\mathbf{M}$, let $\kappa_{2}$ be small. Then, for any $0<\eta<1$, if $\int_{B_{\rho}}|\mathrm{Rm}|^{2} d \mu \leq \kappa_{2}$, we have

$$
\sup _{B_{(1-\eta)^{2+2} \rho}}\left|\nabla^{l} \mathrm{Rc}\right| \leq C\left(\rho, \eta, \kappa_{2}, C_{s}\right) .
$$

PROOF. We have proved the case $l=0$. For $l=1$, firstly, from Lemma 2.4 and integration by parts, we have $\int_{B_{(1-\eta)^{2} \rho}}|\nabla \mathrm{Rc}|^{2} d \mu \leq C(H, V, D, n, \eta, \rho)$.

Now again, integrating by parts, one obtains

$$
\begin{aligned}
\int_{B_{\rho}}\langle & \left.\Delta \nabla \mathrm{Rc}, u^{2} \nabla \mathrm{Rc}|\nabla \mathrm{Rc}|^{2 p-2}\right\rangle d \mu \\
= & -\int_{B_{\rho}} u^{2}|\nabla \mathrm{Rc}|^{2 p-2}\left|\nabla^{2} \operatorname{Rc}\right|^{2} d \mu-\int_{B_{\rho}} u^{2}\left\langle\nabla^{2} \mathrm{Rc}, \nabla \operatorname{Rc} \nabla|\nabla \operatorname{Rc}|^{2 p-2}\right\rangle d \mu \\
& -\int_{B_{\rho}} u \nabla u\left\langle\nabla^{2} \mathrm{Rc}, \nabla \operatorname{Rc}|\nabla \operatorname{Rc}|^{2 p-2}\right\rangle d \mu \\
\leq & -\int_{B_{\rho}} u^{2}\left|\nabla^{2} \operatorname{Rc}\right|^{2}|\nabla \operatorname{Rc}|^{2 p-2} d \mu+2 \int_{B_{\rho}} u|\nabla u|\left|\nabla^{2} \mathrm{Rc} \| \nabla \operatorname{Rc}\right|^{2 p-1} d \mu .
\end{aligned}
$$

This and $2 a b \leq \epsilon a^{2}+b^{2} / \epsilon$ imply

$$
\begin{gathered}
\int_{B_{\rho}} u^{2}\left|\nabla^{2} \mathrm{Rc}\right|^{2}|\nabla \mathrm{Rc}|^{2 p-2} d \mu \leq \int_{B_{\rho}}\left|\left\langle\Delta \nabla \mathrm{Rc}, u^{2} \nabla \mathrm{Rc}|\nabla \mathrm{Rc}|^{2 p-2}\right\rangle\right| d \mu \\
+\epsilon \int_{B_{\rho}} u^{2}\left|\nabla^{2} \mathrm{Rc}\right|^{2}|\nabla \mathrm{Rc}|^{2 p-2} d \mu+\frac{1}{\epsilon} \int_{B_{\rho}}|\nabla \mathrm{Rc}|^{2 p} d \mu,
\end{gathered}
$$

choosing $\epsilon$ small enough, we have

$$
\begin{aligned}
\int_{B_{\rho}} u^{2}\left|\nabla^{2} \operatorname{Rc}\right|^{2}|\nabla R c|^{2 p-2} d \mu \\
\quad \leq \int_{B_{\rho}}\left|\left\langle\Delta \nabla R c, u^{2} \nabla R c|\nabla R c|^{2 p-2}\right\rangle\right| d \mu+\frac{1}{\epsilon(1-\epsilon)} \int_{B_{\rho}}|\nabla R c|^{2 p} d \mu .
\end{aligned}
$$


On the other hand, from Lemma 2.5

$$
\begin{aligned}
\int_{B_{(1-m) \rho}} & \left\langle\Delta \nabla \mathrm{Rc}, u^{2} \nabla \mathrm{Rc}|\nabla \mathrm{Rc}|^{2 p-2}\right\rangle \mid d \mu \\
\leq & C \int_{B_{\rho}} u^{2}\left|\operatorname{Rm}\left\|\nabla^{2} \mathrm{Rc}\right\| \nabla \mathrm{Rc}\right|^{2 p-2} d \mu+\frac{C}{\rho} \int_{B_{\rho}} u|\operatorname{Rm} \| \nabla \mathrm{Rc}|^{2 p-1} d \mu \\
& +C \int_{B_{\rho}} u^{2}|\operatorname{Rm} \| \nabla \mathrm{Rc}|^{2 p} d \mu,
\end{aligned}
$$

which implies

$$
\begin{aligned}
(1-\epsilon) & \int_{B_{(1-n) \rho}} u^{2}\left|\nabla^{2} \mathrm{Rc}\right|^{2}|\nabla \mathrm{Rc}|^{2 p-2} d \mu \\
& \leq \frac{C}{\epsilon(1-\epsilon)} \int_{B_{(1-n) \rho}}|\nabla \mathrm{Rc}|^{2 p} d \mu+C \int_{B_{(1-\eta) \rho}} u^{2}|\mathrm{Rm}||\nabla \mathrm{Rc}|^{2 p} d \mu \\
& +\frac{C}{\rho} \int_{B_{(1-\eta) \rho}} u|\operatorname{Rm}||\nabla \mathrm{Rc}|^{2 p-1} d \mu+\frac{C}{\epsilon} \int_{B_{(1-\eta) \rho}} u^{2}|\mathrm{Rm}|^{2}|\nabla \mathrm{Rc}|^{2 p-2} d \mu .
\end{aligned}
$$

It follows that

$$
\begin{aligned}
& \left(\int_{B_{(1-\eta)^{2} \rho}}\left(u|\nabla \mathrm{Rc}|^{p}\right)^{4} d \mu\right)^{1 / 2} \\
& \leq C\left(\left.\left.\int_{B_{(1-\eta)^{2} \rho}} u^{2}|\nabla| \nabla \mathrm{Rc}\right|^{p}\right|^{2} d \mu+\int_{B_{(1-\eta)^{2} \rho}}|\nabla u|^{2}|\nabla \mathrm{Rc}|^{2 p} d \mu\right) \\
& \leq C \int_{B_{(1-n)^{2} \rho}} u^{2}\left|\nabla^{2} \mathrm{Rc}\right|^{2}|\nabla \mathrm{Rc}|^{2 p-2} d \mu+\frac{C}{\rho^{2}} \int_{B_{(1-n)^{2} \rho}}|\nabla \mathrm{Rc}|^{2 p} d \mu \\
& \leq C \int_{B_{(1-\eta)^{2} \rho}}|\nabla \mathrm{Rc}|^{2 p} d \mu+C \int_{B_{(1-\eta)^{2} \rho}} u^{2}|\mathrm{Rm}|^{2}|\nabla \mathrm{Rc}|^{2 p} d \mu \\
& \leq\left(C+\frac{1}{\epsilon}\right) \int_{B_{(1-n)^{2} \rho}}|\nabla \mathrm{Rc}|^{2 p} d \mu \\
& +C \epsilon\left(\int_{B_{(1-\eta)^{2} \rho}}|\mathrm{Rm}|^{8} d \mu\right)^{1 / 2}\left(\int_{B_{(1-\eta)^{2} \rho}} u^{4}|\nabla \mathrm{Rc}|^{4 p} d \mu\right)^{1 / 2} .
\end{aligned}
$$

From Lemma 2.7, if $\epsilon$ is small enough, we obtain

$$
\left(\int_{B_{(1-\eta)^{4} \rho}}\left(u|\nabla \mathrm{Rc}|^{p}\right)^{4} d \mu\right)^{1 / 2} \leq C \int_{B_{(1-\eta)^{2} \rho}}|\nabla \mathrm{Rc}|^{2 p} d \mu .
$$

Using Moser iteration again, this implies Theorem 2.8 for $l=1$. Then by induction, based on Lemma 2.5 and Lemma 2.7, the theorem follows easily. For details, see Theorem 3.9 of [2]. 
Moreover, we have

$$
\Delta \mathrm{Rm}=\mathrm{Rm} * \mathrm{Rm}+\nabla^{2} \mathrm{Rc}
$$

and

$$
\Delta \nabla^{l} \mathrm{Rm}=\sum_{k=0}^{l} \nabla^{k} \mathrm{Rm} * \nabla^{l-k} \mathrm{Rm}+\nabla^{l+2} \mathrm{Rc}
$$

This implies

PROPOSITION 2.9. ([2, Theorem 3.12.]) For any critical metric $g$ on $M$, let $\kappa_{3}$ be small. Then, for any $0<\eta<1$, if $\int_{B_{\rho}}|\operatorname{Rm}|^{2} d \mu \leq \kappa_{3}$, we have

$$
\sup _{B_{(1-\eta)^{2+2} \rho}}\left|\nabla^{l} \mathrm{Rm}\right| \leq C\left(\rho, \eta, \kappa_{3}, C_{s}\right), \quad l=0,1,2, \ldots
$$

\section{The diameter estimate of the small geodesic sphere}

In this section, we will give the diameter estimate on the small geodesic sphere. Consider the geodesic ball $B_{\rho}\left(x_{0}\right)$; rewrite the metric $g$ as $g=d r^{2}+\sum g_{i j}(r, \theta) d \theta^{i} d \theta^{j}$, where $r=d\left(x, x_{0}\right)$. Then

$$
R_{r r}=g^{i j} R_{i r r y}=-\frac{\partial^{2}}{\partial r^{2}} \ln \sqrt{G}-\frac{1}{4}\left|\frac{\partial}{\partial r} g\right|_{g}^{2},
$$

where $G=\operatorname{det}\left(g_{i j}\right)$. We have the following lemma:

LEMMA 3.1. ([5]) Suppose that, for some positive constants $K, i_{0}$,

(i) $\operatorname{Rc}(g) \geq-K g$, and

(ii) $i_{M} \geq i_{0}>0$.

Then

$$
\int_{0}^{\rho} r^{2}\left|\frac{\partial}{\partial r} g\right|_{g}^{2} d r \leq C\left(K, i_{0}\right) \rho .
$$

It easily follows from Lemma 3.1 that

LEMMA 3.2. ([7, Proposition 1.11]) There exists a positive constant $C\left(K, i_{0}\right)$ such that

$$
e^{-C\left(r_{2} / r_{1}\right)} \bar{g}\left(r_{1}\right) \leq \bar{g}\left(r_{2}\right) \leq e^{C\left(r_{2} / r_{1}\right)} \bar{g}\left(r_{1}\right)
$$

for $0<r_{1}<r_{2} \leq \rho / 2$, where $\bar{g}(r)=\sum g_{i j}(r, \theta) d \theta^{i} d \theta^{j}$ is the induced metric on the geodesic sphere $S_{r}\left(x_{0}\right)$. 
From Lemma 3.2, and the Theorem 4.14. of [5], we obtain

COROLLARY 3.3. ([5]) For $K>0$, there exist a constant $C=C\left(K, i_{0}\right)>0$ such that for any Riemannian manifold $(M, g)$ with

(i) $\operatorname{Rc}(g) \geq-K g$,

(ii) $i_{M} \geq i_{0}$,

and, for small $i_{0} \geq \rho>0$, we have $\operatorname{diam}\left(S_{\rho}\left(x_{0}\right)\right) \leq C \rho$.

\section{Proofs of the theorems}

In this section, we will prove the compactness property of critical metrics on compact Kähler surfaces.

Let $g_{i j}$ be a smooth metric on a closed 4-manifold $M$. The Gauss-Bonnet formula says

$$
8 \pi^{2} \chi(M)=\int_{M}|\mathrm{Rm}|^{2} d \mu-4 \int_{M}|\mathrm{Rc}|^{2} d \mu+\int_{M} R^{2} d \mu .
$$

Thus, if $\int_{M}|\mathrm{Rc}|^{2} d \mu \leq H$, one obtains

$$
\int_{M}|\mathrm{Rm}|^{2} d \mu \leq C(\chi(M), H) .
$$

Now consider the class $W(M)$ of Theorem 1.1, as we proved in Section 2. We have curvature uniformly bounded on the ball with small $L^{2}$ norm of curvature. On the other hand, if the ball $B_{\rho_{0}}(v)$ does not meet the hypothesis of Proposition 2.6, then we still have

LEMMA 4.1.

$$
|\mathrm{Rm}| \leq \epsilon(r) / r^{2}, \quad \epsilon(r) \rightarrow 0 \quad \text { as } \quad r \rightarrow 0,
$$

where $r(x)=\operatorname{dist}(x, v)$.

PROOF. For each small $\rho>0$, define the metric $\tilde{g}=\rho^{-2} g$. We have

$$
|\widetilde{R m}|=\rho^{2}|\mathbf{R m}| \text {. }
$$

Now fix $x_{0}$ with $r\left(x_{0}\right)=\rho<\rho_{0}$; the local Sobolev constant on $\tilde{B}\left(x_{0}, 1\right)$ is still uniformly bounded, that is,

$$
\left(\int_{\tilde{B}} f^{4} d \tilde{\mu}\right)^{1 / 2} \leq C \int_{\tilde{B}}|\tilde{\nabla} f|^{2} d \tilde{\mu}, \quad f \in C_{0}^{\infty}(\tilde{B}) .
$$


Next, consider

$$
\int_{\tilde{B}\left(x_{0}, 1 / 2\right)}|\widetilde{\mathrm{Rm}}|^{2} d \tilde{\mu}=\int_{B\left(x_{0}, \rho / 2\right)}|\mathrm{Rm}|^{2} d \mu .
$$

For $\rho>0$ small, in view of (3), we have $\int_{B\left(x_{0}, \rho / 2\right)}|\mathrm{Rm}|^{2} d \mu \leq \kappa_{1}$, using Proposition 3.1. It follows that

$$
|\widetilde{\mathrm{Rm}}| \leq C(\rho, \kappa, H, \chi(M))
$$

on $\tilde{B}\left(x_{0}, 1 / 2\right)$. From the local Sobolev constant bound, we have, for small $\rho>0$,

$$
\operatorname{Vol}\left(B\left(x_{0}, \rho / 2\right)\right) \geq C \rho^{4}
$$

for $C$ independent of $\rho$. By scale invariance,

$$
\operatorname{Vol}\left(\tilde{B}\left(x_{0}, 1 / 2\right)\right) \geq C
$$

From (6), (7) and (8), one obtains $|\widetilde{R m}| \rightarrow 0$ as $r(x) \rightarrow 0$. This and (4) yield Lemma 4.1.

Set

$$
A(\rho, \bar{\rho})=B_{2 \rho}(v)-B_{\bar{\rho}}(v)=\{x \in M, \bar{\rho}<r(x)<2 \rho\}
$$

Consider $\rho^{-1} A(\rho, \bar{\rho})$ as a Riemannian manifold with $A(\rho, \bar{\rho})$ with the metric $\rho^{-2} g$. Then, by blowing up at this point, from Lemma 4.1, and Corollary 3.3, we have the following theorem:

THEOREM 4.2. ([2, Lemma 5.3]) $\left(\rho^{-1} A(\rho, 0), x_{\rho}\right)$ converges to $\left(D^{4}-\{0\}, e\right)$ in the $C^{2}$-topology, where $D^{4}=\left\{w \in \mathbf{R}^{4} ;|w|<2\right\}$ is the Euclidean ball of radius 2 .

First, from Theorem 4.2, we have $r^{-1} S_{r} \rightarrow S^{3}(1) \subset R^{4}$, where $S^{3}(1)$ is the standard sphere of radius 1 in $R^{4}$. Then, in the Hausdorff metric, we take $r_{o}$ small such that, for $r \leq r_{0}$, we have ([4])

$$
H^{3}\left(r^{-1} S_{r}\right) \leq 2 H^{3}\left(S^{3}(1)\right) \leq C(4)
$$

which implies

$$
H^{3}\left(S_{r}\right) \leq C r^{3}
$$

This implies that

$$
\operatorname{Vol}(S(r)) \leq C r^{3}, \quad \text { for small } r
$$


Then, studying the tangent cone metric at each singular point by blowing up this point, we obtain a complete Riemannian manifold $(\bar{M}, h)$ such that

$$
\begin{aligned}
& \text { (i) } \operatorname{Vol}\left(B_{r}\right) \leq C r^{4} \\
& \text { (ii) }\left.\left|\int_{\bar{M}}\right| \operatorname{Rm}\right|^{2} d \mu<\infty \\
& \text { (iii) }|\operatorname{Rm}(z)|=1 \text {, for some } z \in \bar{M} \\
& \text { (iv) } i \bar{M} \longrightarrow \infty \\
& \text { (v) }|\operatorname{Rm}| r^{2} \longrightarrow 0 \text { as } r \rightarrow \infty .
\end{aligned}
$$

By Proposition 6.1 and Corollary 6.7 of [1], (i), (ii), (iv), (v) of (11) imply this tangent cone metric is the flat Euclidean metric. This leads to a contradiction with (iii) of (11) (cf. Lemma 5.8 and (5.15) of [2]). Hence the curvature tensors of critical manifolds at each singular point are uniformly bound. Then, applying the Gromov compactness theorem, we obtain Theorem 1.1.

On the other hand, from (10), Lemma 4.1 and Theorem 4.2, combining the arguments as in Section 3 and Section 4 of [8], Theorem 1.3 follows easily.

\section{References}

[1] S. Bando, A. Kasue and H. Nakajima, 'On a construction of coordinates at infinity on manifolds with fast curvature decay and maximal volume growth', Invent. Math. 97 (1989), 313-349.

[2] S. C. Chang, 'Critical Riemannian metrics', Math. Z. (to appear).

[3] A. Derdziński, 'Self-dual Kähler manifolds and Einstein manifolds of dimension four', Compositio Math. 49 (1983), 405-433.

[4] H. Federer, Geometric measure theory (Springer, Berlin, 1969).

[5] L. Zhiyong Gao, 'Einstein metrics', J. Differential Geom. 32 (1990), 155-183.

[6] — , 'Convergence of Riemannian manifolds; Ricci pinching and $L^{n / 2}$-curvature pinching', $J$. Differential Geom. 32 (1990), 349-381.

[7] — ' ' $L^{n / 2}$-Curvature pinching', J. Differential Geom. 32 (1990), 731-774.

[8] G. Tian, 'On Calabi's Conjecture for complex surfaces with positive first Chern class', Invent. Math. 101 (1990), 101-172.

[9] — , 'Compactness theorems for Kähler-Einstein manifolds of dimension 3 and up', J. Differential Geom. 35 (1992), 535-558.

Department of Mathematics

National Tsing Hua University

Hsinchu

Taiwan 30043 